\title{
Managing Wireless Sensor Networks with Supply Chain Strategy
}

\author{
${ }^{\dagger}$ Wei Liu,${ }^{\dagger}$ Yanchao Zhang, ${ }^{\ddagger}$ Wenjing Lou, and ${ }^{\dagger}$ Yuguang Fang \\ ${ }^{\dagger}$ Department of Electrical and Computer Engineering \\ University of Florida \\ Gainesville, FL 32611 \\ Email: \{liuw@,yczhang@,fang@ece.\}ufl.edu \\ $\ddagger$ Department of Electrical and Computer Engineering \\ Worcester Polytechnic Institute \\ Worcester, MA 01609 \\ Email:wjlou@ece.wpi.edu
}

\begin{abstract}
Wireless sensor networks (WSNs) are appealing in obtaining fine-granular observations about the physical world. Due to the fact that WSNs are composed of a large number of low-cost but energy-constrained sensor nodes, along with the notorious timer-varying and error-prone natures of wireless links, scalable, robust, and energy-efficient data disseminating techniques are requisite for the emerging WSN applications such as environment monitoring and surveillance. In this paper we examine this emerging field from a view of supply chain management and propose a hybrid data dissemination framework for WSNs. In particular, we conceptually partition a whole sensor field into several functional regions based on the supply chain management methodology, and apply different routing schemes to different regions in order to provide better performance in terms of reliability and energy usage. For this purpose, we also propose a novel zone flooding scheme, essentially a combination of geometric routing and flooding techniques. Our hybrid data dissemination framework features low overhead, high reliability, good scalability and flexibility, and preferable energy efficiency. Detailed simulation studies are carried out to validate the effectiveness and efficiency of our scheme.
\end{abstract}

\section{INTRODUCTION}

Recent advances in MEMS technology and wireless communications have resulted in small, low-cost sensors with more and more powerful processing and networking capabilities. This makes wireless sensor networks (WSNs) be identified as one of the most important technologies that will change the world [1]. WSNs can furnish us with fine-granular observations about the physical world where we are living. Potential applications include disaster rescue, energy management, medical monitoring, logistics and inventory management, and military reconnaissance, etc. While much research has focused on making sensor networks feasible and useful [2] [3], some important problems resulting from the error-prone and resourceconstrained natures of WSNs are not well addressed yet. Of note are the issues associated with scalability, reliability, and network lifetime. For instance, a data dissemination technique should work well not only in small-scale sensor networks but also in large-scale sensor networks. In addition, it should be robust against harsh environmental effects and temporal or permanent failures of sensors and wireless links in between them. Moreover, it should have good energy-efficiency in terms of both low average energy consumption per observation report and balanced energy usage instead of overusing a small set of the network.
This paper is targeted for real-time and continuous monitoring applications such as battlefield monitoring networks and volcano monitoring networks, where networking sensors are deployed in an ad hoc manner and the aforementioned nice properties are desirable. Those sensors collaboratively accomplish the sensing task and forward the sensing data to the closest data processing centers or sink nodes through multihop wireless links. Traditional routing protocols proposed for ad hoc networks are unsuitable for our target applications owing to the substantial differences between ad hoc networks and sensor networks pointed out in [2]. In contrast, flooding, as a reactive technique with inbred reliability, seems to be a good candidate for sensor networks because it does not involve costly topology maintenance and complex route discovery algorithms. However, the main problems with flooding are that it typically causes unproductive and often harmful bandwidth congestion, as well as inefficient use of node resources such as energy, which is scarce in resource-constrained sensor networks. Though several data dissemination schemes have been proposed specifically for sensor networks [2] [3], the research on finding a good scheme that is able to strike a good balance among reliability, scalability and energy efficiency is still thin on the ground.

In this paper, we propose a hybrid data dissemination framework for WSNs that features low overhead, high reliability, good scalability and flexibility, and preferable energy efficiency. Our contributions in this paper are mainly threefold. First of all, to the best of our knowledge, this is the first effort to study a wireless sensor network from a view of supply chain management. We introduce the notion of supply chain into the design of sensor networks and conceptually partition a sensor field into several functional regions according to supply chain management methodology. Secondly, we apply different routing techniques to different regions in order to provide better performance in terms of reliability and fair energy usage. Lastly, we propose a novel zone flooding scheme which is a combination of flooding and geometric routing techniques. Our rationale here is to offer the desirable reliability and routing simplicity that is associated with flooding and to mitigate the deficiency of blind flooding with geometric routing.

The remainder of this paper is structured as follows. We start with discussing some basics of supply chains and the resemblances between supply chains and wireless sensor networks 


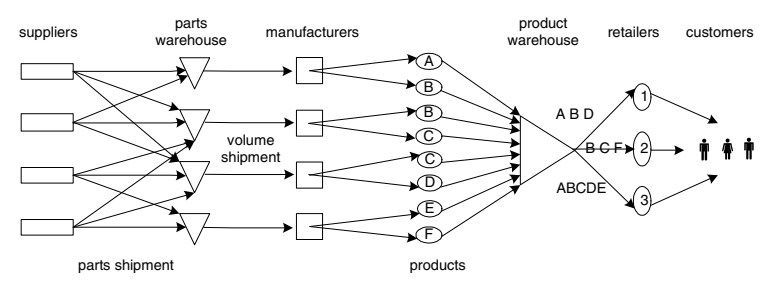

Fig. 1. An exemplary supply chain.

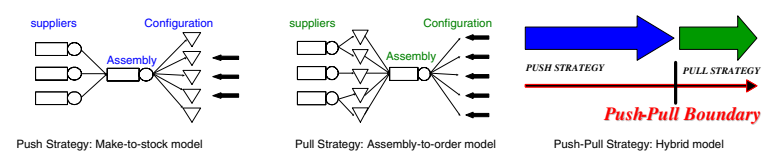

Fig. 2. Supply chain strategies.

in Section II. Then we detail our hybrid data dissemination framework in Section III. In Section IV, simulation studies are carried out to evaluate the performance of our scheme. Finally the concluding remarks are given in Section V.

\section{Modelling Sensor Networks as Supply Chains}

\section{A. Introduction to Supply Chains}

In the business world, supply chain (SC) is the series of links and shared processes existing between suppliers and customers, which involve all activities from the acquisition of raw materials to the delivery of finished goods to end consumers. Fig. 1 shows an exemplary SC, where raw materials or parts enter into a manufacturing organization via a supply system and are transformed into finished goods. The finished goods are then supplied to consumers through a distribution system.

In the past 40 years SC strategies have evolved from push strategies to pull strategies and finally to push-pull strategies [9]. In a push-type SC, production and distribution decisions are based on long-term forecasts. The manufacturer uses orders received from the retailer warehouses to forecast demands. And consumers' order decisions are based on inventory rather than consumers' demand. In contrast, in a pull-type SC, which is hard to leverage economies of scale, production and distribution are demand-driven and are based on actual customer demands rather than forecasts. In hybrid push-pull strategies, push strategies are applied in the initial stages such as parts inventory, where production and distribution decisions are based on long-term demand forecasts by manufacturers on the basis of orders received from retailers; and pull strategies take effect in the final stages such as product assembly, where production and distribution are purely demand driven and rely on actual customer demands rather than forecasts. Usually a buffer inventory, such as warehouse, is located at the push-pull boundary. Fig. 2 shows those three different strategies.

On the other hand, supply chain management (SCM), a vital factor for competitive advantage and sustainable business improvement, is the act of optimizing all activities throughout the supply chain, so that products and services are supplied in the right quantity, to the right location, at the right time, and at the optimal cost. One of the fundamental concepts in SCM is
TABLE I

THE CORRESPONDENCE TABLE BETWEEN SUPPLY CHAINS AND SENSOR NETWORKS

\begin{tabular}{|l|l|}
\hline Items in supply chains & Counterpoints in sensor networks \\
\hline Raw materials or parts & Phenomena of interest \\
\hline Suppliers or manufacturers & Sensor nodes generating the data \\
\hline Transportation network & Intermediate sensor nodes \\
\hline Distributors or retailers & Sink nodes \\
\hline Finished products & Data processed by sink nodes \\
\hline Consumers & End-users of the data offered by sink nodes \\
\hline
\end{tabular}

that all the autonomous entities in the SC may have their own inner operations and management strategies, but they work in a cooperative fashion to achieve the management goal of the whole SC: satisfying the customer service requirements while minimizing the overall system cost and obtaining as many revenue as possible from the cooperation.

\section{B. How can supply chains help us?}

Supply chains and wireless sensor networks have many key components or functions in common. For example, the parts warehouse in Fig. 1 is designed to consolidate raw parts from different suppliers and decouple need from availability, and its counterpart in sensor networks is the desirable functionality of "data aggregation", which is used to combine the data coming from different sources enroute with the aim of eliminating redundancy, minimizing the number of transmissions, and thus saving energy [4]. Besides, the shown product warehouse close to retailers, ensuring low inventory, reduced transportation costs, and quick replenishment capability, acts the similar roles to those of the mechanisms for reducing information implosion caused by flooding in sensor networks. In addition, vital to the cost management of supply chains, the transportation planning is equivalent in functionality to the routing in sensor networks, whose major objective is to transfer sensing events from sensors to sink nodes as efficiently as possible. Moreover, both require all the entities in the system to work cooperatively for goals common in nature: providing good QoS and keeping the overall system cost as small as possible.

Interestingly, we notice that two notable routing protocols for sensor networks, namely, directed diffusion [5] and SPIN [6], accord well with the SC methodology. In particular, directed diffusion can be viewed as a pull-type SC, in which the sink node propagates its interests throughout the sensor network and sensors satisfying the interests respond with sensing data via intermediate sensors. Thus, directed diffusion is a pull-type $\mathrm{SC}$ in that production is demand (interest) driven. In contrast, SPIN with ADV-REQ-DATA handshaking is more like a push-type SC in that it is designed for disseminating information to all nodes in a sensor network, where the nodes generating the data can be regarded as suppliers or manufacturers in a SC. Thus, SPIN is a push-type SC where order decisions (REQ) are based on inventory (ADV).

The above resemblance between supply chains and sensor networks motivates us to model a WSN as a SC and thus we can utilize the knowledge of SCM in the business world 


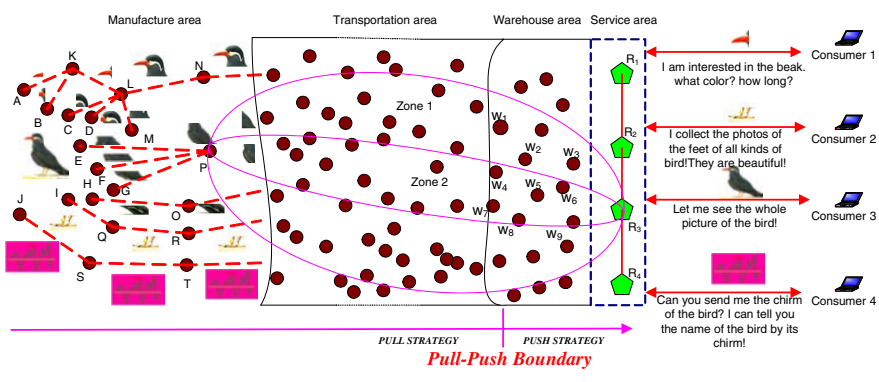

Fig. 3. System architecture for habitat monitoring.

to improve the performance of the sensor network. More specifically, we can utilize the ideas of partitioning the supply chain into several different components (partition strategy), applying different management mechanisms to different components (hybrid strategy), and designing cooperations among different components (cooperation strategy) to solve our sensor networking problem. Table I shows the correspondence between supply chains and sensor networks, in which intermediate nodes sometimes are also called "semi-manufacturers" or "parts warehouses" when performing data aggregation to reduce the data redundancy. We emphasize here that the aforementioned classification of sensors is only conceptual and each sensor may act as different roles under different scenarios. For example, one sensor may be a "supplier" of one communication, while be a "transporter" of the other communication in the meantime.

\section{A HYBRID DATA DiSSEMINATION FRAMEWORK FOR SENSOR NETWORKS}

\section{A. System model}

In what follows, we use a WSN for habitat monitoring to illustrate our scheme. As shown in Fig. 3, we divide the whole sensor field into several functional areas according to the aforementioned push-pull strategies (see Section II-A) for supply chains. In the manufacture area, some nodes such as those from $A$ to $\mathrm{J}$, are involved in generating the raw data about the objects of interest, i.e., birds in this case, while other nodes such as $K, L$, and $\mathrm{P}$ are responsible for data aggregation, i.e., consolidating the raw data and reducing the possible information overlap. The filtered data is fed into the transportation area to be collaboratively relayed by intermediate sensors to sink nodes. In addition, we introduce the warehouse area as a buffer area between the transportation area and the service area to reduce the possible traffic congestion and information implosion [2] at the sink nodes. The service area consists of sink nodes which can directly communicate with each other through fast and reliable links, either wired or wireless. The sink nodes perform collaborative reception of sensing events and offer different data items to end-users or consumers with different interests.

We assume that each node has knowledge of its own position and of the positions of the sink nodes. We further assume all the nodes including common sensor nodes and sink nodes are identified by their geographic information. The location of the aforementioned manufacture area is not fixed in that any node sensing the events of interest can form a manufacture area together with its neighboring nodes. So does the transportation area lying in the forward direction from the manufacture area towards the sink nodes. In this paper we assume the sinks are far enough away from the manufacture area, thus transportation area can exist. In contrast, the service area is determinate because of the invariable locations of sink nodes. Moreover, we define the warehouse area to be the area within sink nodes' $n$-hop ranges, where $n$ is a tunable design parameter. To form its warehouse area, one sink node just needs to broadcast a special request with the TTL value set to $n$. Any node receiving this request becomes a warehouse member (warehouse node) of the warehouse area of the requesting sink node.

One of the novel features of our hybrid data dissemination paradigm is that we apply different data forwarding mechanisms to different functional areas, which are elaborated in the following sections.

\section{B. Manufacture area}

We assume that the nodes in the manufacture area are aware of their own missions. Each mission might represent a sensing task of the sensor network. In this example, the mission may be collecting the information of birds, such as the beak color, the feet length, or even the bird chirms. Due to the limitation of sensors' capabilities, each sensor may only sense part of the interested event so that they might need to locally exchange some sensing events and select one of them as an aggregation center to fulfill the data fusion task. For example, nodes $K$, $L$, and $P$ in Fig. 3 are selected as aggregation centers. Since in most cases aggregation centers are only several hops away from the sensing nodes, the simplest way to forward the sensed raw data to aggregation centers is to broadcast packets with limited TTL values. For lack of space, we do not detail how to manage the sensing tasks and accomplish data aggregation in this paper.

Besides data fusion, each aggregation center assumes a special role in our data dissemination framework. It needs to determine the transportation method for the filtered data, i.e., using single zone flooding or multi-zone flooding, and the proper transportation zone(s) through which the data will travel in the transportation area. For example, after finishing the aggregation of the raw data from nodes $E, F$, and $G$, node $P$ makes the choice of using two flooding zones and then chops the filtered data into two parts, both of which are labelled with their respective designated flooding zone.

In fact, the operations in manufacture area show lots of flexibility during the selection of the transportation method and the flooding zone. If the environment is good, an aggregation center can choose a single flooding zone to forward the data. In addition, if no warehouse area is allocated and the flooding zone is the whole sensor field, our zone flooding will reduce to the pure flooding. On the other hand, if we can specify the flooding zone only containing a single path, our zone flooding will reduce to the single path routing. For a bursty and 


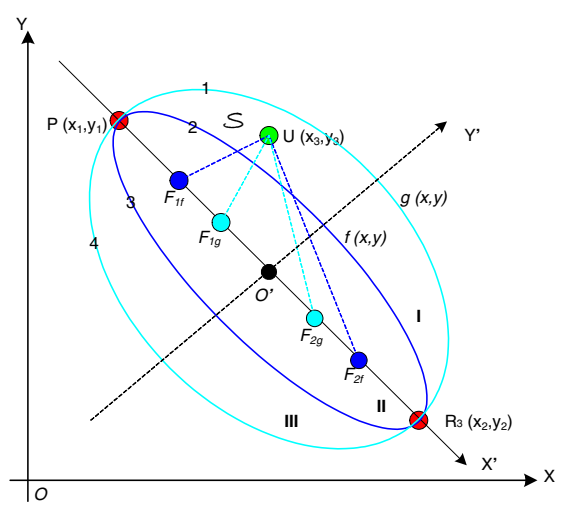

Fig. 4. The forwarding-decision-making process of nodes in the transportation area.

bulky event report, an aggregation center can choose multiple flooding zones and chop the report into several portions and simultaneously deliver them to multiple sinks in different flooding zones. Since sink nodes can communicate with each other through fast and reliable links, they can exchange the received portions and easily reconstruct the original event report. Such multipath approaches are also well known for improving the reliability and security of data disseminations [7]. Moreover, to combat node or wireless link failures, an aggregation center may introduce some redundancy by sending duplicate reports to the sinks in multiple zones. We should note that, aggregation centers can change the sizes and the locations of flooding zones with time to evenly distribute the traffic load among sensors and to avoid overusing a small set of nodes. Besides, with proper scheduling, nodes may wake/sleep zone by zone or in other manners to save energy.

\section{Transportation area}

Sensor nodes in the transportation area undertake the task of relaying data to sink nodes. To avoid costly topology maintenance and complex route discovery algorithms, we propose a novel zone flooding scheme, which is a combination of geometric routing and flooding techniques. The basic idea is as follows: once a node receives a packet carrying parameters that identify a flooding zone, it first needs to determine whether it is in the indicated zone or not through some trivial calculations using its own location information and the received zone parameters. Only when situated in the flooding zone could it rebroadcast the packet.

Fig. 3 shows an example our zone flooding scheme, in which ellipses are used to specify the flooding zones. Suppose one of the aggregation centers, say node $P$, has the coordinates $\left(x_{1}, y_{1}\right)$ in the cartesian plane of Fig. 4, and the intended sink node $R_{3}$ has the coordinates $\left(x_{2}, y_{2}\right)$. Besides the filtered data, each data packet sent from node $P$ will carry four extra zone parameters: AC_location indicating the coordinates of the aggregation node $P$, sink_location indicating the coordinates of sink node $R_{3}$, and Inner-SemiminorAxis and OuterSemiminorAxis used to denote the semiminor of the inner and outer ellipses of the desired flooding zone respectively.
Accordingly, when one node, say $U$ with coordinates $\left(x_{3}, y_{3}\right)$, receives such a data packet, the question whether it should rebroadcast the packet or not can be reduced to the simple geometric problem: whether does point $U$ lie between two ellipses determined by end points of the major-axis $P$ and $R_{3}$, and the Inner-SemiminorAxis $b_{1}$ and the Outer-SemiminorAxis $b_{2}$ ? Suppose the semiminor axis of an ellipse with two majoraxis endpoints $P$ and $R_{3}$ is $b$. Then the sum of the distance from point $U$ to two fixed points $F_{1}$ and $F_{2}$ (the foci) can be expressed as $L(b)=D_{1}+D_{2}$ [12], where

$D_{1}=\sqrt{\left(\sqrt{\frac{\left(x_{1}-x_{2}\right)^{2}+\left(y_{1}-y_{2}\right)^{2}}{4}-b^{2}}+\left(x_{3}-\frac{x_{1}+x_{2}}{2}\right)\right)^{2}+\left(y_{3}-\frac{y_{1}+y_{2}}{2}\right)^{2}}$

and

$D_{2}=\sqrt{\left(\sqrt{\frac{\left(x_{1}-x_{2}\right)^{2}+\left(y_{1}-y_{2}\right)^{2}}{4}-b^{2}}-\left(x_{3}-\frac{x_{1}+x_{2}}{2}\right)\right)^{2}+\left(y_{3}-\frac{y_{1}+y_{2}}{2}\right)^{2}}$.

Therefore, node $U$ needs to rebroadcast the packet after checking $L\left(b_{1}\right)<2 a<L\left(b_{2}\right)$ for $b_{1} \neq b_{2}$, or $L\left(b_{1}\right)=L\left(b_{2}\right)<2 a$ for $b_{1}=b_{2}$, where $a=$ $\sqrt{\left(x_{1}-x_{2}\right)^{2}+\left(y_{1}-y_{2}\right)^{2}} / 2$ is the semimajor axis of the ellipse with major-axis endpoints $P$ and $R_{3}$. Otherwise, it will simply ignore the packet because it is not in the specified flooding zone for that packet. Following the above procedures, sensor nodes ("transporters") in the transportation area can finally relay the data to the warehouse area through multi-hop wireless links.

We notice that two ellipses with the same endpoints can jointly determine six different flooding zones (see Fig. 4). To avoid the possible confusion about which zone should be used, we adopts a simple rule by using the positive value of the semiminor axis, e.g., $b_{1}$ or $b_{2}$, to denote the half part of an ellipse close to the positive direction in the shifted coordinate plane, while using the negative values of the semiminor axis to denote the half part close to the negative direction in the shifted coordinate plane. For example, $b_{1}$ together with $b_{2}$ determines the zone I, while $-b_{1}$ and $-b_{2}$ jointly specify the zone III. In fact, by varying the values of semiminor axis, we can easily get physically separated or interleaved multiple paths (multiple flooding zones) without incurring any additional costs. As discussed in section III-B, the multipath routing is a powerful technique to improve the reliability and other system performance.

Fig. 5 shows the typical structure of a data packet "manufactured" by an aggregation node. The field "Warehouse Flag" is used to inform intermediate nodes whether this packet has ever been processed by a warehouse node. The usage of this field will be discussed in section III-D. In addition, based on the fields "Interest (event) Description" and "Other Control Fields", intermediate nodes and sinks can determine whether one received packet have already been processed, or assemble those partitioned packets belonging to the same interests, or remove the possible redundancy added to improve the data transmission reliability.

In our example, since we use two ellipses to specify one flooding zone, the "Flooding Zone Parameters" field only 


\begin{tabular}{|c|c|c|}
\hline AC_location & Sink_location & Flooding Zone Parameters \\
\hline $\begin{array}{c}\text { Interest (event) Description } \\
\text { (location, time, type, ...) }\end{array}$ & Warehouse Flag & $\begin{array}{c}\text { Other Control Fields } \\
\text { (multiple-zone flooding, } \\
\text { redundancy, ...) }\end{array}$ \\
\hline \multicolumn{2}{|c}{ Payload } \\
\hline
\end{tabular}

Fig. 5. An exemplary packet structure.

needs to include one inner semiminor axis and one outer semiminor axis. In fact, any two noncrossing curves sharing the same two ends could be used to specify a flooding zone. Nevertheless, we should choose those curves that not only can be represented with as few bytes as possible to reduce the communication overhead, but also can simplify the forwarding-decision-making processes of intermediate nodes. In this sense, arcs and ellipses are two promising candidates. Moreover, a flooding zone specified by two curves should be large enough to have sufficient nodes to forward the packets while maintaining high energy efficiency in the meantime. To accomplish this, the aggregation center in our example, say node $P$, should properly choose the values of the semiminors of the two ellipses. Besides, to balance the nodal usage in the transportation area, aggregation centers should change the flooding zones by alternatively using different negative and positive values of semiminor axis. Therefore, our scheme achieves the even load distribution and the fair energy usage without incurring any additional costs.

\section{Warehouse area and service area}

In the previous exemplary SC shown in Fig. 1, the warehouse near the retailer creates a break point in the movement of the goods and acts as a buffer to reduce the cost of stock at the retailer, hence improving the flexibility of the retailer. The warehouse frequently updates its inventory list to the retailers, and the retailers can quickly get the products out of stock in the store replenished from the warehouse. We notice that such a warehouse component is also needed in sensor networks for realtime and continuous monitoring applications. In those applications, bursty and bulky traffic may be simultaneously transmitted to sink nodes, as a result of which notorious traffic congestion may happen frequently in the vicinity of sink nodes and thus cause the unfavorable loss of information and the waste of scarce network resources. Moreover, the redundant packets flooded towards sinks may result in the information implosion problem as well. Thus, the introduction of the warehouse area as a buffer area can decouple need (interests) from availability (redundant event reports) and help mitigate the above information implosion problem and possible traffic congestion.

For the warehouse area, we use a modified SPIN [6] instead of the zone flooding as the underlying routing protocol. Different from the ADV-REQ-DATA exchange in SPIN that happens between neighbors, the ADV-REQ-DATA exchange in our scheme is executed with unicasting between a warehouse node and a sink node several hops away. The unicasting path

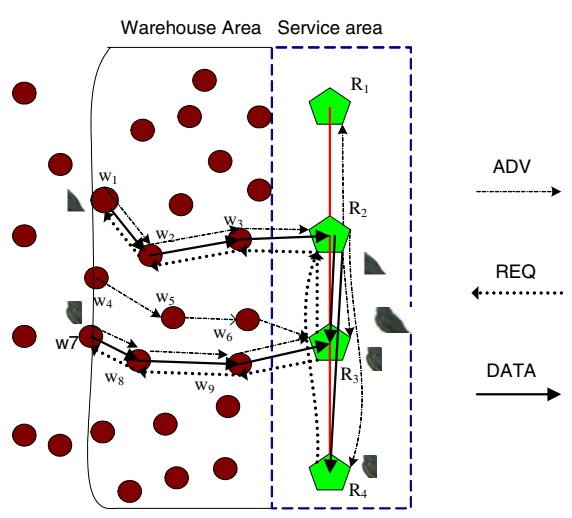

Fig. 6. The routing process in the warehouse area.

could be established using DSDV [8]. We note that, only those warehouse nodes may participate in the routing maintenance activities. With a limited warehouse size, small overhead of routing maintenance can be expected. Of course, other routing schemes are applicable to this area as well. Fig. 6 illustrates our routing strategy used in warehouse area. Once receiving data packets from sensor nodes out of the warehouse area (by examining the field "Warehouse Field"), a sensor node, say $W_{7}$ lying in both the flooding zone and the warehouse area, first sets the field "Warehouse Flag" and temporarily stores those packets. Afterwards, $W_{7}$, the data holder, will unicast an ADV message, essentially an inventory containing the descriptors of stored packets, to the targeted sink node $R_{3}$, either on a perpacker basis or periodically or when the number of stored packets exceeds a threshold. After that, $R_{3}$ will send a REQ message requesting for the interested data. Once seeing the REQ message, $W_{7}$ can unicast the requested data to $R_{3}$ via a DATA message. In case that $R_{3}$ does not receive the requested data in time after sending out a REQ, it can resend a REQ to the same data holder $W_{7}$ or another data holder who also sent to it an ADV containing the descriptors of the same data.

Here we want to explain how the warehouse area can help reduce the information implosion. Suppose packets describing the same event for sink $R_{3}$ arrive at $W_{4}$ and $W_{7}$, respectively, which are situated in the same flooding zone and the warehouse area of $R_{3}$. Using the above procedure, both $W_{4}$ and $W_{7}$ will send ADV messages to $R_{3}$. It is up to $R_{3}$ to make a decision on which one should send the data based on some criterions such as hop counts or delay. Suppose $W_{7}$ is chosen, $R_{3}$ will send a REQ to $W_{7}$ and accordingly $W_{7}$ can unicast the requested data via a DATA message to $R_{3}$. After a certain period, $W_{4}$ may delete the stored stale data. We can see that redundant packets can be successfully eliminated by the means of ADV-REQ-DATA exchanges.

Moreover, sink nodes in the service area perform collaborative reception in the sense that they could communicate with each other through fast and reliable means, e.g., wired links or separate wireless channels. For example, if sink $R_{2}$ receives an ADV message from node $W_{1}$, it can contact other sink nodes far from $W_{2}$ to see if they need the provided data, 


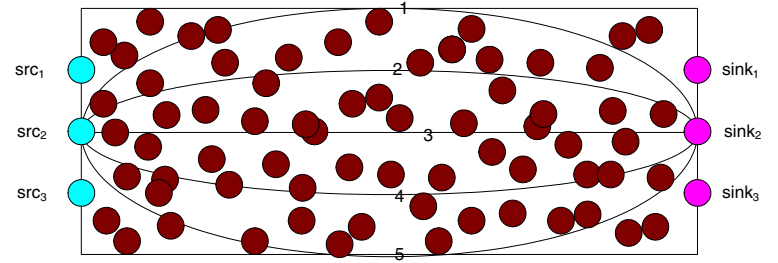

Fig. 7. The simulated sensor field.

TABLE II

Simulation CONFIGURATION

\begin{tabular}{|c|c|}
\hline Simulation Area & $500 \mathrm{~m} \times 300 \mathrm{~m}$ \\
\hline Number of Nodes & 606 \\
\hline Transmission Range & $40 \mathrm{~m}$ \\
\hline Initial Energy & $60 \mathrm{~J}$ \\
\hline Transmit Power & $81 \mathrm{~mW}$ \\
\hline Receive/Idle Power & $30 \mathrm{~mW}$ \\
\hline Radio Bandwidth & $2 \mathrm{Mbps}$ \\
\hline Data packet & 128 Bytes \\
\hline Directed Diffusion interests & 36 Bytes \\
\hline ADV/REQ & 12 Bytes \\
\hline
\end{tabular}

though $R_{2}$ itself may not need it. Suppose $R_{4}$ needs the data, $R_{2}$ can help obtain the data from $W_{1}$ and sends it to $R_{4}$. Such collaboration also helps a warehouse node deal with the case when the warehouse node has no unicasting route to the desired sink of the report due to node failures or other reasons. In such a case, the warehouse node may choose to send the ADV message to a sink closest to itself.

\section{Performance Evaluation}

\section{A. Methodology and Metrics}

To evaluate our proposed scheme (denoted by RRP), we have developed an evaluation environment within Glomosim [10] and implemented our hybrid data dissemination paradigm, including the zone flooding scheme. We simulate a sensor field consisting of 606 sensor nodes. We have 3 independent equally-spaced sources at the left boundary of the sensor field and 3 independent equally-spaced sinks at the right boundary of the sensor field. The other 600 sensors are uniformly deployed in the sensor field. The sensor field topology is shown in Fig. 7, where the sensor field is composed of the transportation area and the warehouse area only, while the manufacture area and the service area are on the boundaries of the field. Besides, each of three sources generates a 128byte data packet (event) destined to a random sink every 1.5 seconds, 1.0 second, and 2.0 seconds, respectively. To study the performance of the zone flooding scheme and routing in the warehouse area alone, there is no data fusion and collaboration among sink nodes in our simulation. Therefore, a source acts as both an event observer and a data aggregation center. Table II lists the configuration parameters of our simulation, where the transmission/reception power consumption of sensors are in line with those of Motes [11].

In our simulation, we vary the size of the warehouse area, defined as the area within a sink node' $n$-hop range, to see its impact on RRP's performance. Besides, each source-sink pair uses $d$ (another control parameter) equally-spaced elliptic curves to specify the flooding zones and these curves can closely span the whole sensor filed. Fig. 7 shows the curves used by source $s r c_{2}$ and $s i n k_{2}$ where $d=4$. And $s r c_{2}$ always chooses two consecutively numbered curves to specify a flooding zone. Furthermore, we introduce an environment parameter, namely, packet error rate $(P E R)$, to reflect the error-prone natures of wireless links in WSNs. For simplicity, we assume all radio transmissions are independent but have the identical PERs during one simulation run. Therefore, by varying the control parameters $n$ and $d$, and $P E R$, we can study the performance of our proposed RRP in different settings.

We compare our RRP with pure flooding and directed diffusion [5] in terms of energy efficiency and reliability. There are four performance metrics of interest to us. The event delivery ratio (EDR) reflecting the reliability is defined as the ratio of the delivered event reports (data packets) to those generated by the sources. The normalized energy consumption is defined as the total energy consumed for the transmission and reception of data and routing packets in the simulation time, which is normalized by the energy consumed for one single data reception and averaged over all data packets and the number of nodes. The average event end-to-end delay is defined as the average delay from when a packet (event) is generated and transmitted by the source till it is received by the sink. And the average routing overhead is defined as the routing packets generated in the simulation time averaged over all transmitted data packets.

\section{B. Simulation results}

For the first set of figures (Fig. 8(a) - Fig. 8(d)), we set $d$ to be 4 , i.e., $d=4$, and study the performance of RRP under different $(P E R)$ and $n$.

Fig. 8(a) compares the event delivery ratio of our RRP, pure flooding and directed diffusion under different PERs. As we can see, since directed diffusion maintains single path for each source-sink pair, its EDR is very sensitive to the change of PER, dropping almost linearly from $99 \%$ to $86 \%$ with the increase of PERs. In contrast, the EDRs of pure flooding always stabilize around $100 \%$. This result is not surprising because of the inbred reliability of flooding techniques. In addition, our RRP always demonstrates a stable EDR greater than $99 \%$ under all five different warehouse sizes. Therefore, by using zone flooding in the transportation area and unicasting in the warehouse area, our RRP has the reliability comparable to that of flooding, but superior to that of directed diffusion.

Fig. 8(b) shows the normalized energy consumption of our RRP, pure flooding and directed diffusion. Compared with the other schemes, directed diffusion demonstrates the minimum energy consumption because it uses low rate flooding for interest propagation and unicasting for data packets. In addition, our RRP outperforms pure flooding because of the use of zone flooding instead of network-wide flooding. Moreover, 


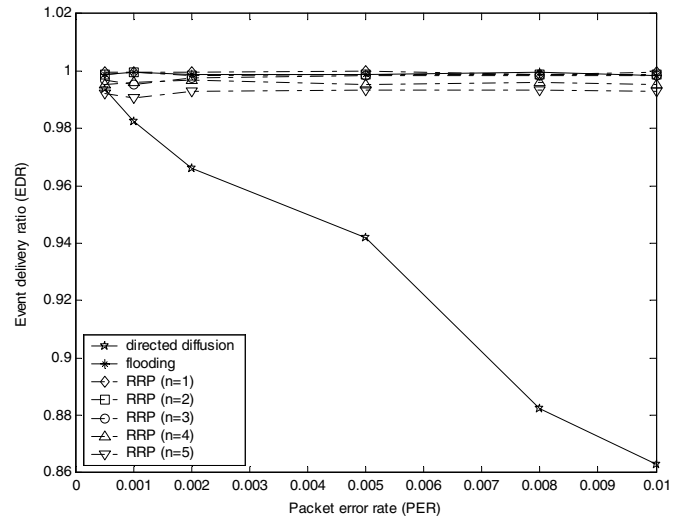

(a) Event delivery ratio vs. packet error rates.

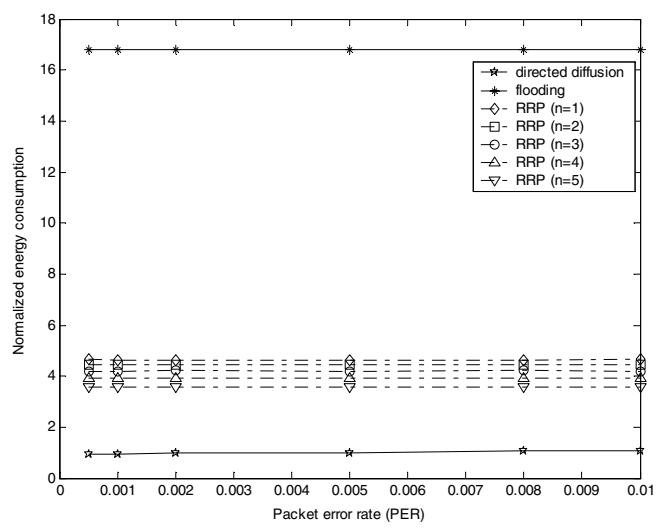

(b) Normalized energy consumption vs. packet error rates.

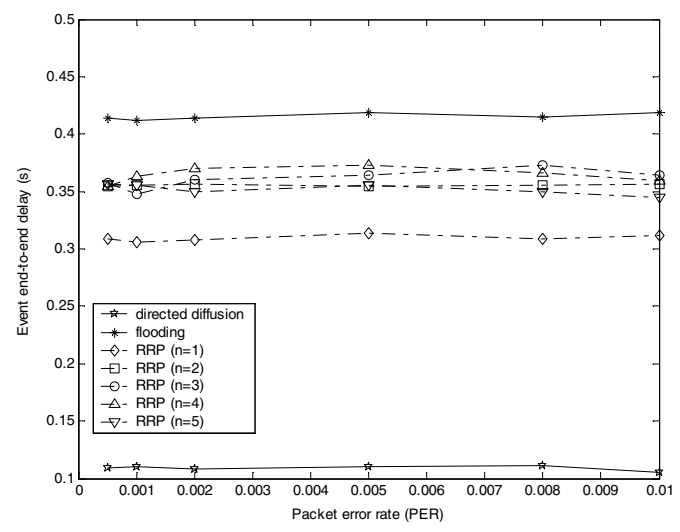

(c) Average event end-to-end delay vs. packet error rates.

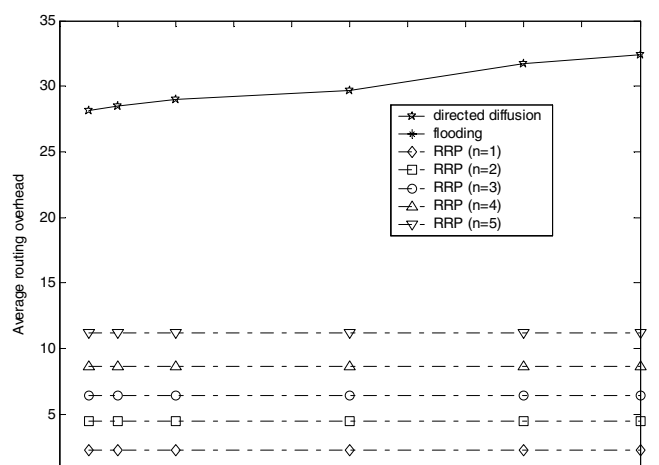

Proceedings of the First International C.onference on 0 uality of Service in Heterogeneous Wired/Wireless Networks (QSHINE'04) 0-7695-2233-5/04 \$20.00 @ 2004 IEEEErate (PER) we observe that the greater $n$ is, the less energy our RRP consumes. The reason is that zone flooding and unicasting are respectively used in the transportation area and the warehouse area. With the increase of $n$, more unicasting and less zone flooding will be involved and the former is known to be more energy efficient than the latter.

Fig. 8(c) gives the average event end-to-end delay of each scheme under different PERs. Since directed diffusion adopts minimum-delay paths, it has the shortest event delay among the three compared schemes. For pure flooding, the networkwide pure flooding of data packets may result in much more collisions than our zone flooding and packets belonging to the same source-sink pair may follow different, quite unpredictable, and possibly very long routes. Therefore, pure flooding demonstrates longer average event delay than that of our RRP. In addition, for our RRP, since an event report should first travel through the transportation area and then the warehouse area, there are two sources contributing delays. One is zone flooding in the transportation area, and the other is ADV-REQ-DATA exchanges in the warehouse area. Moreover, the average delay coming from zone flooding decreases with the increase of $n$, while the average delay coming from ADVREQ-DATA exchanges increases with the increase of $n$. Thus, with the alternation of the main source between these two due to the change of $n$, the performance of our RRP is quite different. This phenomenon suggests a tradeoff between zone flooding and warehouse routing should be made to achieve a desired latency.

Fig. 8(d) presents the average routing overhead of each scheme. Among the three schemes, directed diffusion requires sinks to periodically flood interests to maintain the gradients, as a result of which it displays the largest routing overhead. For our RRP, the routing overhead comes from the routing maintenance in the small warehouse area. Therefore, it has larger routing overhead than that of pure flooding which is assumed to have zero routing overhead. We can also observe the overhead of RRP increases with the increase of $n$.

For the second set of figures (Fig. 9 - Fig. 10), we fix $n$ to be 3 , i.e, $n=3$, and study the performance of RRP under different $P E R$ and $d$.

Fig. 9 shows the event delivery ratio with different $d$. We observe that the size of flooding zone has obvious impact on the performance of RRP. When $d=2$, the EDR of RRP is nearly $98 \%$, but when $d$ increases to 3 or 4 , the EDR of RRP becomes better and is comparable to that of pure flooding. However, the larger $d$ does not always imply better EDR. As we can see, when $d$ is 5 , the EDR drops to $96 \%$. This phenomenon can be interpreted as follows. When $d$ is small, the collisions in the zone may occur quite often and result in relatively lower EDR. Though pure flooding suffers from collisions as well, flooding has higher probability to receive a copy of the original data packet because it is flooded in the whole field rather than in a zone. With the increase of $d$ and thus the decrease of the flooding zone size, packet collisions may decrease, leading to a better EDR. However, the flooding zone size should be reasonably large to have enough nodes to

(d) Average routing overhead vs. packet error rates.

Fig. 8. Simulation results of RRP with different $n$ 


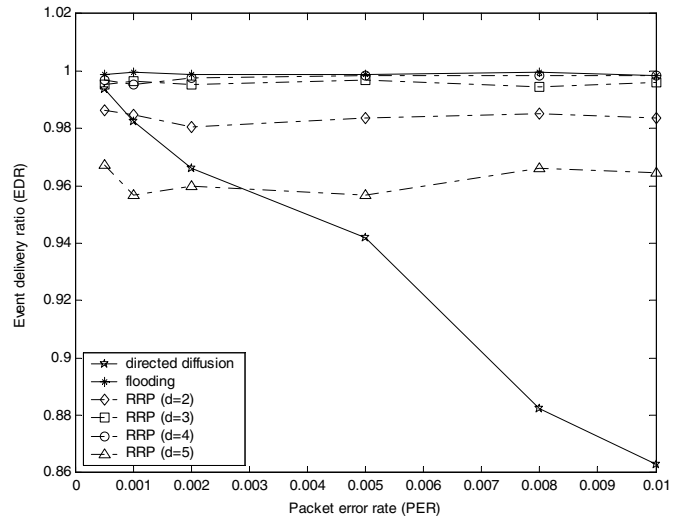

Fig. 9. Event delivery ratio vs. packet error rates.

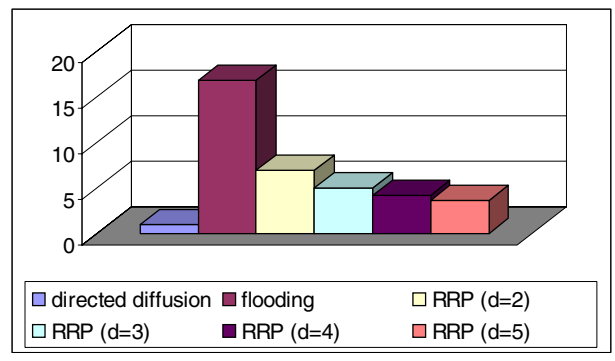

(a) Normalized energy consumption vs. $d$ (PER=0.0005).

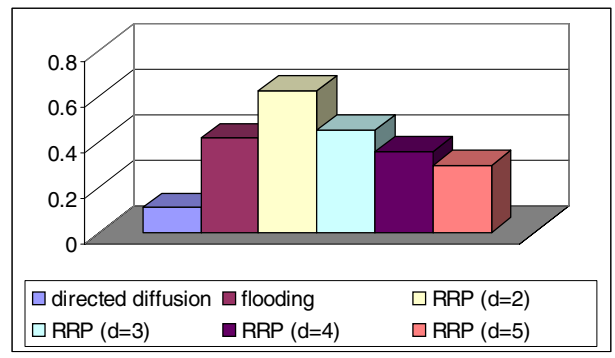

(b) Average event end-to-end delay vs. $d(\mathrm{PER}=0.0005)$.

Fig. 10. Energy consumption and event end-to-end delay of RRP with different $d$

relay packets. That's the reason why we observe EDR drops to $96 \%$ when $d$ is 5 ! Furthermore, our RRP outperforms directed diffusion in most cases even when the flooding zone is small.

Fig. 10(a) shows the normalized energy consumption of our RRP with different $d$, where $P E R=0.0005$. The results of pure flooding and directed diffusion are shown for reference only. We observe that the larger $d$ is, the less energy each packet consumes. Considering the impact of $d$ on both EDR and energy consumption, we clearly see that the flooding zone size should be well chosen to strike a balance between reliability and energy efficiency.

Fig. 10(b) shows the average event end-to-end delay of our RRP with different $d$, where $P E R=0.0005$. From Fig. 10(b), we can see that the latency of RRP decreases with the increase of $d$. When $d$ is small, packets received by a sink may travel along unpredictable paths, leading to a longer average delay. In contrast, When $d$ is large, meaning a small flooding zone and fewer nodes involved in the packet forwarding, packets received by a sink may travel along more predictable paths limited by the small zone, which results in a shorter average delay.

To sum up, in terms of reliability, $P E R$ has smaller impact on our RRP and pure flooding than on directed diffusion. And by choosing different warehouse sizes and flooding zone sizes, we can adjust the normalized energy consumption, average event end-to-end delay, and the average routing overhead of RRP to acceptable values while maintaining nearly perfect reliability.

\section{CONClusion}

In this paper we introduce the concept of supply chain into wireless sensor networks and propose a hybrid data dissemination framework based on the supply chain management methodology. We conceptually partition a whole sensor field into several functional regions and apply different routing schemes to different regions in order to provide better performance in terms of reliability and fair energy usage. For this purpose, we also propose a novel zone flooding technique, which is a combination of geometric routing and flooding techniques. The effectiveness and efficiency of our scheme are validated through simulation studies. Our hybrid data dissemination framework features low overhead, high reliability, good scalability, and notable flexibility.

\section{ACKNOWLEDGMENT}

This work was supported in part by the ONR under grants N000140210464 and N000140210554.

\section{REFERENCES}

[1] 10 emerging technologies that will change the world. Technology Review, vol.106, no.1, pp. 33-49, Feb. 2003.

[2] I. Akyildiz, W. Su, Y. Sankarasubramaniam, and E. Cayirci. A survey on sensor networks. IEEE Communication Magazine, August 2002

[3] C-Y Chong and S. P. Kumar. Sensor networks: evolution, opportunities, and challenges. Proceedings of the IEEE, vol.91, no.8, August 2003.

[4] B. Krishnamachari, D. Estrin, and S. Wicker. Impact of Data Aggregation in Wireless Sensor Networks. Proc. of International Workshop on Distributed Event-Based Systems, Vienna, Austria, July 2002.

[5] C. Intanagonwiwat, R. Govindan, and D. Estrin. Directed Diffusion: a scalable and robust communication paradigm for sensor networks. Proc. of the Sixth Аnииal ACM/IEEE International Conference on Mobile Computing and Networking (MobiCom00), Boston, Aug. 2000.

[6] J. Kulik, W. R. Heinzelman, and H. Balakrishnan. Negotiation-based protocols for disseminating information in wireless sensor networks. ACM Wireless Networks, vol. 8, no. 2-3, pp. 169-185, 2002.

[7] W. Lou, W. Liu, and Y. Fang. SPREAD: enhancing data confidentiality in mobile ad hoc networks. Proc. of IEEE INFOCOM04, HongKong, China, Mar 2004.

[8] C. E. Perkins and P. Bhagwat. Highly dynamic destination-sequenced distance-vector routing (DSDV) for mobile computers. Com. Commun. Rev., pp. 234-44, Oct. 1994.

[9] L. D. Fredenhall and E. Hill. Basics of supply chain management, St. Lucie Press, 2001.

[10] L. Bajaj, M. Takai, R. Ahuja, K. Tang, R. Bagrodia, and M. Gerla. GloMoSim: A Scalable network simulation environment, UCLA Computer Science Department Technical Report 990027, May 1999.

[11] http://www. xbow. com.

[12] E. W. Weisstein. "Ellipse" From MathWorld-A Wolfram Web Resource. http: //mathworld.wolfram. com/Ellipse.html. 